\title{
REGRESSÃO LINEAR COM DUPLO TRUNCAMENTO NA DISTRIBUIÇÃO DA VARIAYVLL DEPENDENTE (1)
}

Do problema do ajuste de uma regressão linear, quando a distribuição da variável dependente tem duplo truncamento, utilizando a função de máxima verossimilhança e um processo iterativo.

\section{$1-$ INTRODUCAO}

Motivados pela necessidade de estudar o comportamento da idade da mulher casada na época do primeiro abôrto em função da sua idade ao casar (Milanesi ", 1968), ou seja, estabelecer a regressão entre estas variáveis, nos defrontamos com um problema envolvendo uma variável que, pela sua própria natureza, possui um campo de variação restrito a um sub-conjunto do total. Estamos nos referindo à variável - idade da mulhér casada na época do primeiro abôrto que tem seu campo de variação limitado, de um lado, pela idade ao casar, e de outro, pela idade na menopausa, ou seja, tem o que se denomina um duplo truncamento.

- A solução do problema proposto se enquadra, portanto, no capítulo de regressão com duplo truncamento na distribuição da variável dependente. HoL. GATE $^{2}$ (1965) já considerou o caso de regressão com truncamento simples; o presente trabalho representa uma extensão dos resultados daquele autor, para o caso mais geral de duplo truncamento.

\section{2 - A FUNCAO DENSIDAdE DE PROBABILIdAdes}

No que segue faremos abstração da motivação já apresentada, a fim de tratarmos do problema com maior generalidade.

Recebido para publicação em 16-12-1968.

(1) Da Cadeira de Estatistica Aplicada à Saúde Pública da Faculdade de Higiene e Saúde Pública da USP. 
SOUZA, J. M. P. de — Regressão linear com duplo truncamento na distribuição da variável dependente. Rev. Saúde públ., S. Paulo, 2(1):1-9, Jun. 1968.

Sejam $x$ a variável independente e $y$ a variável aleatória dependente distribuída normalmente, com média $a+b x$ e variância $\sigma^{2}$, ou, abreviadamente:

$$
y \div N\left(a+b x ; \sigma^{2}\right)
$$

Havendo duplo truncamento, a função densidade de probabilidde de $y$, para cada $x$ é:

$$
f(y / x)=\frac{1}{J \sqrt{2 \pi}} e^{-\frac{1}{2}\left(\frac{y-b x}{0}\right)^{2}} \cdot \frac{1}{I(w, z)}
$$

onde

$$
\begin{gathered}
I(w, z)=\int_{\frac{w \cdot a \cdot b x}{\sigma}}^{\frac{z \cdot a \cdot b x}{\sigma}} \frac{1}{\sqrt{2 \pi}} e^{-\frac{t^{2}}{2}} d t \\
w \leq y \leq z ;
\end{gathered}
$$

ou seja, w e $z$ são os pontos inferior e superior de truncamento na distribuição de $y$.

\section{3 - EstimaÇa dos PARÁmetros a E b DA RETA DE REGREsSAo \\ E DA VARIANCIA $\sigma^{2}$}

A estimação será feita pelo método da máxima verossimilhança. Para tanto, suponhamos que, em correspondência a cada $x_{i}(i=1,2, \quad m)$ fôsse tomada uma amostra de tamanho $n_{i}$, isto é, de valores de $y_{i j}\left(j=1,2, \ldots, n_{i}\right)$. Sejam:

$$
\sum_{i=1}^{m} \quad n_{i}=N, \quad-\frac{a}{a}=\xi, \quad-\frac{b}{\sigma}=k .
$$


SOUZA, J. M. P. de - Regressão linear com duplo truncamento na distribuicão da variável dependente. Rev. Saúde públ., S. Paulo, 2(1):1-9, jun. 1968.

A função de verossimilhança da amostra é:

$$
I=\prod_{i=1}^{m} \prod_{j=1}^{n_{i}} \frac{1}{\sigma \sqrt{ } 2 \pi} e^{-\frac{1}{2}\left(\frac{y_{i j}}{\sigma}+\xi+k x_{i}\right)^{2}} \frac{1}{I\left(w_{i}, z_{i}\right)}
$$

Tomando-se logaritmos naturais, temos:

$$
\begin{aligned}
\log L=L^{*}=-N \log \sigma & -\frac{N}{2} \log 2 \pi-\frac{1}{2} \sum_{i=1}^{m} \sum_{j=1}^{n_{i}}\left(\frac{y_{i j}}{\sigma}+\xi+k x_{i}\right)^{2}- \\
& -\sum_{i=1}^{m} n_{i} \log I\left(w_{i}, z_{i}\right)
\end{aligned}
$$

Calculando-se as derivadas parciais para cada parâmetro, obtemos:

$$
\begin{aligned}
& \partial_{L^{*}}=f(\xi)=-\frac{\sum_{i=1}^{m} \sum_{j=1}^{n_{i}} y_{i j}}{\sigma}-N \xi-k \sum_{i=1}^{m} n_{i} x_{i}+\sum_{i=1}^{m} n_{i} \frac{e\left(w_{i}\right)-e\left(z_{i}\right)}{I\left(w_{i}, z_{i}\right)} \\
& \frac{\partial L^{*}}{\partial k}=g(k)=-\frac{\sum_{i=1}^{m} \sum_{j=1}^{n_{i}} y_{j} x_{i}}{\sigma}-\xi \sum_{i=j}^{m} n_{i} x_{i}-k \sum_{i=1}^{m} n_{i} x_{i}^{2} \sum_{i=j}^{m} n_{i} x_{i} \frac{e\left(w_{i}\right)-e\left(z_{i}\right)}{I\left(w_{i}, z_{i}\right)}
\end{aligned}
$$

$$
\begin{aligned}
& \frac{\partial L^{*}}{\partial \sigma^{\prime}}=h(\sigma)=-\frac{N}{\sigma}+\frac{\sum_{i=1}^{m} \sum_{j=1}^{n_{i}} y_{i j}^{2}}{\sigma^{3}}+\frac{\xi}{\sigma^{2}} \sum_{i=1}^{m} \sum_{j=1}^{n_{i}} y_{i j}^{2}+\frac{k}{\sigma^{2}} \sum_{i=1}^{m} \sum_{j=1}^{n_{i}} x_{i} y_{i j}+ \\
& +\frac{1}{\sigma^{2}} \sum_{i=1}^{m} n_{i} \frac{w_{i} \cdot e\left(w_{i}\right)+z_{i} \cdot e\left(z_{i}\right)}{I\left(w_{i}, z_{i}\right)}
\end{aligned}
$$

onde $e\left(w_{i}\right), e\left(z_{i}\right)$ são as ordenadas obtidas na curva normal, nos pontos de truncamento $w_{i}$ e $z_{i}$.

Derivando-se uma segunda vez, olotemos: 


$$
\begin{aligned}
& \frac{\partial f}{\partial \xi}=\sum_{i=1}^{m} n_{i} \frac{I_{\left(w_{i}, z_{i}\right)}\left[E_{\left(z_{i}\right)} \cdot e_{\left(z_{i}\right)}-E_{\left(w_{i}\right)} \cdot e_{\left(w_{i}\right)}\right]+\left[e_{\left(z_{i}\right)}\right] 2+\left[e_{\left(w_{i}\right)}\right]^{2}-2\left[e_{\left(z_{i}\right)} \cdot e_{\left(w_{i}\right)}\right]}{\left[I_{\left.\left(w_{i}, z_{i}\right)\right]^{2}}\right.}-N \\
& \frac{\partial f}{\partial k}=\frac{\partial g}{\partial \xi}=\sum_{i=1}^{m} n_{i} x_{i} \frac{\left.I_{\left(w_{i}, z_{i}\right)}\left[\bar{z}_{\left(z_{i}\right.}\right) \cdot e\left(z_{i}\right){ }^{-E}\left(w_{i}\right) \cdot e\left(w_{i}\right)\right]+\left[e\left(z_{i}\right)\right]^{2}+\left[e_{\left(w_{i}\right)}\right]^{2}-2\left[e_{\left(z_{i}\right)} \cdot e\left(w_{i}\right)\right]}{\left[I_{\left.\left(w_{i}, z_{i}\right)\right]^{2}}^{2}\right.}-\sum_{i=1}^{m} n_{i} x_{i}
\end{aligned}
$$

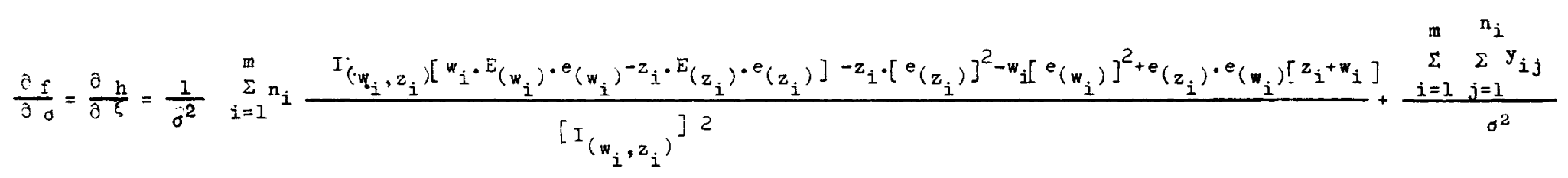

$$
\begin{aligned}
& \frac{\partial g}{\partial k}=\sum_{i=1}^{m} n_{i} x_{i}^{2} \frac{\left.I_{\left(w_{i}, z_{i}\right)}\left[E_{\left(z_{i}\right.}\right)^{(e}\left(z_{i}\right)^{-e}\left(w_{i}\right)\right]+\left[e_{\left(z_{i}\right)}\right]^{2}+\left[e\left(w_{i}\right)\right]^{2}-2\left[e_{\left(z_{i}\right)} \cdot e\left(w_{i}\right)\right]}{\left[{ }^{I}\left(w_{i}, z_{i}\right)\right]^{2}}-\sum_{i=1}^{m} n_{i} x_{i}^{2} \\
& \frac{\partial g}{\partial}=\frac{\partial h}{\partial k}=\frac{1}{\sigma^{2}} \sum_{i=1}^{m} n_{i} x_{i} \frac{\left.I_{\left(w_{i}, z_{i}\right)}\left[w_{i} \cdot E_{\left(w_{i}\right)} \cdot e_{\left(w_{i}\right)}-z_{i} \cdot E_{\left(z_{i}\right.}\right) \cdot e\left(z_{i}\right)\right]-z_{i} \cdot\left[e_{\left(z_{i}\right)}\right]^{2}-w_{i}\left[e_{\left(w_{i}\right)}\right]^{2}+e_{\left(z_{i}\right)} \cdot e\left(w_{i}\right)\left[z_{i}+w_{i}\right]}{\left[I_{\left(w_{i}, z_{i}\right)}\right]^{2}}+\frac{\sum_{i=1}^{m} \sum_{j=1}^{n_{i}} y_{i j} x_{i}}{\sigma^{2}}
\end{aligned}
$$




$$
\begin{aligned}
& \frac{\partial h}{\partial \partial}=\frac{N}{\sigma^{2}}-\frac{2}{\sigma^{3}} \sum_{i=1}^{m} n_{i} \frac{z_{i} \cdot e\left(z_{i}\right)}{I_{\left(w_{i}, z_{i}\right)}}+\frac{1}{\sigma^{4}} \sum_{i=1}^{m} n_{i} \cdot z_{i} \frac{I_{\left(w_{i}, z_{i}\right)}\left[E_{\left(z_{i}\right)} \cdot e_{\left(z_{i}\right)} \cdot z_{i}\right]+\left[e_{\left(z_{i}\right)}\right] 2 \cdot z_{i}-w_{i} \cdot e\left(z_{i}\right) \cdot e\left(w_{i}\right)}{\left.\left[w_{i}, z_{i}\right)\right]^{2}}- \\
& -\frac{2}{\sigma^{3}} \sum_{i=1}^{m} n_{i} \frac{w_{i} \quad e_{\left(w_{i}\right)}}{I_{\left(w_{i}, z_{i}\right)}}+\frac{1}{\sigma^{4}} \sum_{i=1}^{m} n_{i} w_{i} \frac{I_{\left(w_{i}, z_{i}\right)}\left[E_{\left(w_{i}\right)} \cdot e_{\left(w_{i}\right)} \cdot w_{i}\right]-\left[e_{\left(w_{i}\right)}\right]^{2} \cdot w_{i}+z_{i} \cdot e_{\left(z_{i}\right)} \cdot e_{\left(w_{i}\right)}}{\left[I_{\left(w_{i}, z_{i}\right)}\right]^{2}}- \\
& -\frac{1}{\sigma^{4}}\left[3 \sum_{i=1}^{m} \sum_{j=1}^{n_{i}} y_{i j}^{2}+20 \xi \sum_{i=1}^{m} \sum_{j=1}^{n_{i}} y_{i j}+20 k \sum_{i=1}^{m} \sum_{j=1}^{n_{i}} y_{i j} x_{i}\right]
\end{aligned}
$$


SoUZA, J. M. P. de - Regressão linear com duplo truncamento na distribuicão da variável dependente. Rev. Saúde públ., S. Paulo, 2(1):1-9, jun. 1968.

onde:

$$
\begin{aligned}
& \mathrm{F}_{\left(\mathrm{w}_{1}\right)}=\frac{\left(\mathrm{w}_{\mathrm{i}}\right)}{\sigma}+\xi+k \mathbf{x}_{\mathrm{i}} \\
& \mathrm{F}_{\left(\mathrm{z}_{\mathrm{i}}\right)}=\frac{\left(\mathrm{z}_{\mathrm{i}}\right)}{\sigma}+\xi+k \mathbf{x}_{\mathrm{i}} .
\end{aligned}
$$

Em virtude da dificuldade de resolver estas equações de forma explícita, os estimadores $\hat{a}, \hat{b}, \hat{\sigma}$, , serão obtidos a partir dos resultados numéricos das cquaçōes 1 a 9, por processo iterativo, utilizando-se então o método de NewtonRaphson (WhitTaKer \& RoBinson ${ }^{7}, 1926$ ).

Como primeiro passo, calculam-se valores $\xi_{1}, \hat{k}_{1}$ e $\hat{\sigma}_{1}$, pelo processo clássico de regressão e análise de variância, e, com êstes valores, resolve-se o seguinte sistema de 3 equações a 3 incógnitas:

$$
\begin{aligned}
& \mathbf{f}(\xi)+\mathrm{p} \frac{\partial \mathrm{f}}{\partial \xi}+\mathbf{q}-\frac{\partial \mathrm{f}}{\partial \mathrm{k}}+\mathrm{r}-\frac{\partial \mathrm{f}}{\partial \boldsymbol{\sigma}}=\mathbf{0} \\
& \mathrm{g}(\mathbf{k})+\mathbf{p} \frac{\partial \mathrm{g}}{\partial \xi}+\mathrm{q} \frac{\partial \mathrm{g}}{\partial \mathbf{k}}+\mathrm{r} \frac{\partial \mathrm{g}}{\partial \boldsymbol{\sigma}}=0 \\
& \mathbf{h}(\boldsymbol{\sigma})+\mathbf{p} \frac{\partial \mathbf{h}}{\partial \xi}+q \frac{\partial \mathbf{h}}{\partial \mathrm{k}}+\mathrm{r} \frac{\partial \mathbf{h}}{\partial \sigma}=0,
\end{aligned}
$$

onde as derivadas são tomadas nos pontos $\xi=\hat{\xi}_{1} ; k=\hat{k_{1}}$ e $\sigma=\hat{\sigma_{1}}$, obtendo-se valores de $p_{1}, q_{1}, r_{1}$, respectivamente, os acréscimos de $\hat{\xi}_{1}, \hat{k}_{1}$ e $\hat{\sigma}_{1}$ na $1 .{ }^{\text {a }}$ iteração. Assim, passamos a ter os seguintes novos estimadores de $\xi, k$ e $\sigma$ :

$$
\begin{aligned}
& \hat{\xi}_{2}=\hat{\xi}_{1}+\mathrm{p}_{1} \\
& \hat{\mathrm{k}}_{2}=\hat{\mathrm{k}}_{1}+\mathrm{q}_{1} \\
& \stackrel{\Lambda}{\sigma_{2}}=\hat{\sigma}_{1}+\mathrm{r}_{1}
\end{aligned}
$$

Os resultados de $11,12,13$ é que serão utilizados agora num $2 .^{\circ}$ ciclo interativo para solução do sistema 10 (onde as derivadas serão tomadas nos pontos $\xi={ }_{\xi_{2}}^{\wedge} ; k=\hat{k}_{2} ; \sigma={\stackrel{\Lambda}{\sigma_{2}}}_{2}$ ) e obtenção de novos acréscimos e, portanto, novos (stimadores. $O$ processo se encerra quando os acréscimos $\left(p_{n}, q_{n}, r_{n}\right)$ se tornarem desprezíveis, e então:

$$
\hat{\xi}_{n+1}=\hat{\xi}_{n} ; \hat{k}_{n+1}=\hat{k}_{n} ; \hat{\sigma}_{n+1}=\hat{\sigma}_{n}
$$


SoUZA, J. M. P. de - Regressão linear com duplo truncamento na distribuição áa variável dependente. Rev. Saúde públ., S. Paulo, 2(1):1-9, Jun. 1968.

Nestas condições, os estimadores de $a, b$ e $\sigma$ serão:

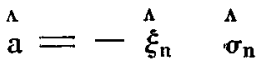

$$
\begin{aligned}
& \hat{\jmath}=-\hat{k}_{\mathfrak{n}} \quad \hat{\sigma}_{\mathrm{n}} \\
& \hat{\sigma}=\sigma_{\mathfrak{n}}, \quad \text { e a reta de regressão: } \\
& \stackrel{\hat{Y}}{\mathrm{i}}_{\mathbf{i}}=\hat{\mathrm{a}}+\hat{\mathrm{b}} \mathbf{x}_{\mathrm{i}} \text {. }
\end{aligned}
$$

\section{4 - CONSIDERACOES SOBRE OS RESULTADOS}

Nossos resultados (equações 1 a 9) diferem dos de Holgate (equações 3 e 4) devido ao têrmo $v(x)$, que é o quociente da ordenada no ponto de truncamento pela área à sua direita; no caso em aprêço temos truncamento, e êst: fato nos conduz a um quociente em que temos, no numerador, a diferença entre as ordenadas dos pontos de truncamento, e no denominador, a área entre os 2 pontos de truncamento.

Como consequiência, temos lambém as divergências devidas ao têrmo $\lambda(x)=v^{\prime}(x)$, para nós substituido por equaçōes mais complexas.

Essas diferenças não são de todo eliminadas, mesmo quando um dos pontos de truncamento está muito afastado da média, determinando uma ordenada pràticamente igual a zero.

Assim sendo, ainda nestes casos mais favoráveis, não podemos nos utilizar das tabelas devidas a SAmpfond ${ }^{6}$ (1952), que nos dão valores de $v(x)$ e $\lambda(x)$, e devemos recorrer às tabelas da curva normal para áreas e ordenadas (PEARson \& HaRTLEy $^{5}, 1958$ e Bol. $\left.{ }^{1}, 1947\right)$.

$$
5-\text { EXEMPLO }
$$

Retomemos o problema que motivou a generalização aqui apresentada, isto é, estabelecer a reta de regressão entre a "idade da mulher na época do primeiro abôrto" $(y)$ e a "idade da mulher ao casar" $(x)$; MrlanEs ${ }^{3}$ (1968), obteve, pelo método dos mínimos quadrados, $\hat{Y}_{1}=12,46+0,696 x ;{ }^{\wedge} \boldsymbol{\sigma}_{1}=4,90$, observando 306 mulheres casadas que tinham tido abôrto.

Como a distribuição da primeira variável pode ser considerada duplamente truncada, foi utilizado, a seguir, o método aqui descrito para a devida correção, encontrando-se os seguintes valores para as equações 1 a 9 :

$$
\begin{aligned}
& \mathrm{f}(\xi)=47,556 \\
& \mathrm{~g}(\mathrm{k})=1.047,251 \\
& \mathrm{~h}(\boldsymbol{\sigma})=\quad 97,262 \\
& \frac{\partial \mathrm{f}}{\partial \xi}=-231,653 \\
& \frac{\partial \mathrm{f}}{\partial \mathrm{k}}=-4.690,929
\end{aligned}
$$


SOUZA, J. M. P. de - Regressão linear com duplo truncamento na distribuicão da variável dependente. Rev. Saúde puibl., S. Paulo, 2(1):1-9, jun. 1968.

$$
\begin{aligned}
& \frac{\partial \mathrm{f}}{\partial \sigma}=279,302 \\
& \frac{\partial \mathrm{g}}{\partial \mathrm{k}}=-236.276,135 \\
& \frac{\partial \mathrm{g}}{\partial \sigma}=5.844,054 \\
& \frac{\partial \mathrm{h}}{\partial \sigma}=-484,819
\end{aligned}
$$

com a nova equação sendo:

$$
\hat{\mathrm{Y}}_{2}=12,31+0,655 \mathrm{x} ; \quad \hat{\sigma}_{2}=4,61 .
$$

Êstes resultados são muito semelhantes aos anteriores, indicando que não se fazia necessário um nôvo ciclo iterativo.

Usando o método de estimação da máxima verossimilhança, a matriz de variância-covariância dos estimadores de $\xi, k$ e $\sigma$ será dada por (Mood \& GRAYBILL ${ }^{4}$, 1963) :

$$
\Delta_{(\xi, \hat{k}, \hat{k}, \hat{k})}=\left[\begin{array}{c}
-\frac{\partial \mathrm{f}}{\partial \xi}-\frac{\partial \mathrm{f}}{\partial \mathrm{k}}-\frac{\partial \mathrm{f}}{\partial \sigma} \\
-\frac{\partial \mathrm{f}}{\partial \mathrm{k}}-\frac{\partial \mathrm{g}}{\partial \mathrm{k}}-\frac{\partial \mathrm{g}}{\partial \sigma} \\
-\frac{\partial \mathrm{f}}{\partial \sigma}-\frac{\partial \mathrm{g}}{\partial \sigma}-\frac{\partial \mathrm{h}}{\partial \sigma}
\end{array}\right]-1
$$

Denotando por $\left.D_{(a, b,}^{\hat{A}}, \hat{\sigma}\right)$ a matriz de variância-covariância dos estimadores de $a, b$ e $\sigma$, e por $J$ a matriz de transformação:

$$
\mathbf{J}=\left[\begin{array}{rrr}
-\hat{\boldsymbol{\sigma}} & 0 & -\hat{\boldsymbol{\xi}} \\
0 & -\hat{\boldsymbol{\sigma}} & -\hat{\mathbf{k}} \\
0 & 0 & 1
\end{array}\right]
$$

tem-se :

$$
\mathrm{D}_{(\hat{\mathrm{a}}, \hat{\mathrm{b}}, \hat{b})}=\mathrm{J} \Delta \mathrm{J}^{\prime}
$$


SOUZA, J. M. P. de - Regressão linear com duplo truncamento na distribuicão da variável dependente. Rev. Saúde públ., S. Paulo, 2(1):1-9, Jun. 1968.

que no exemplo considerado assumem os seguintes valores:

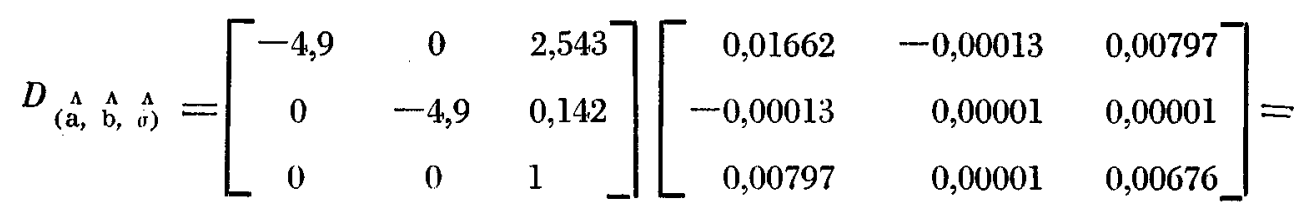

$=\left[\begin{array}{ccc}-4,9 & 0 & 0 \\ 0 & -4,9 & 0 \\ 2,543 & 0,142 & 1\end{array}\right]=\left[\begin{array}{rrr}0,24403 & -0,00640 & -0,02187 \\ -0,00640 & 0,00029 & 0,00092 \\ -0,02187 & 0,00092 & 0,006757\end{array}\right]$,

onde vemos que a variância de $\hat{a}=0,24403$ e a de $\hat{b}=0,00029$, enquanto que a de $\stackrel{\Lambda}{\sigma}=0,00657$.

\section{S U M M A R Y}

A solution to the problem of fitting a linear regression with double truncation in the distribution of the dependent variable is obtained, using the maximum likelihood function and an iterative process.

\section{REFERENCIAS BIBLIOGRAFICAS}

1. BOLI, M. - Tables numériques universelles: des laboratoires et bureaux d'etude. Paris, Dunod, 1947.

2. HOLGATE, P. - Fitting a straight line to data from a truncated population. Biometrics 21(3):715-720, Sept. 1965.

3. MILANESI, M. L. - Abôrto provocado. São Paulo, 1968. (Tese de doutoramento Fac. Hig. Saúde Públ. Univ. S. Paulo).

4. MOOD, A. M. \& GRAYBILL, F. A. - Introduction to the theory of statistics. 2nd ed. New York, Mc-Graw-Hill, 1963. p. 236-237.

5. PEARSON, E. S. \& HARTLEY, H. O., ed. - Biometrika tables for statisticians. 2nd ed. Cambridge, University Press, 1958. v. 1.

6. SAMPFORD, M. R. - The estimation of response-time distributions. II. Multistimulus distributions. Biometrics, 8(4):307-369, Dec. 1952.

7. WHITTAKER, F. T. \& ROBINSON, G. - The calculus of observations: a treatese on numerical mathematics. 2nd ed. London, Blackie, 1926. 


\section{E R R A T A}

Rev. Saúde públ., S. Paulo, 2(1), jun. 1968

Página 2, equação da $6^{\mathrm{a}}$ linha: - corrija-se para

$$
f(y / x)=\frac{1}{\sigma \sqrt{2 \pi}}-\quad e^{-\frac{1}{2}\left(\frac{y-a-b x}{\sigma}\right)^{2}} \frac{1}{I(w, x)}
$$

Página 7, $9^{\circ}$ linha: - onde se lê "no caso em aprêço temos truncamento", leia-se, "no caso em aprêço temos duplo truncamento"

Página 9: - na equação matricial $D_{(a ̂, ~}^{b}, \hat{a}_{)}$, não há o sinal de igualdade entre a $2^{\mathrm{a}}$ e a $3^{\mathrm{a}}$ matriz.

Página 71, 1" linha: - onde se lê "duas a quatro semanas" leia-se "dois a quatro meses" 
O arquivo disponível sofreu correções conforme ERRATA publicada no Volume 2 Número 2 da revista. 